\title{
Sequence-specific validation of LAMP amplicons in real-time optomagnetic detection of Dengue serotype 2 synthetic DNA
}

Minero, Gabriel Khose Antonio; Nogueira, Catarina; Rizzi, Giovanni; Tian, Bo; Fock, Jeppe; Donolato, Marco; Strömberg, Mattias; Hansen, Mikkel Fougt

\section{Published in:}

Analyst

Link to article, DOI:

10.1039/c7an01023k

Publication date:

2017

Document Version

Peer reviewed version

Link back to DTU Orbit

Citation (APA):

Minero, G. K. A., Nogueira, C., Rizzi, G., Tian, B., Fock, J., Donolato, M., Strömberg, M., \& Hansen, M. F. (2017). Sequence-specific validation of LAMP amplicons in real-time optomagnetic detection of Dengue serotype 2 synthetic DNA. Analyst, 142, 3441-3450. https://doi.org/10.1039/c7an01023k

\section{General rights}

Copyright and moral rights for the publications made accessible in the public portal are retained by the authors and/or other copyright owners and it is a condition of accessing publications that users recognise and abide by the legal requirements associated with these rights.

- Users may download and print one copy of any publication from the public portal for the purpose of private study or research.

- You may not further distribute the material or use it for any profit-making activity or commercial gain

- You may freely distribute the URL identifying the publication in the public portal 


\title{
Sequence-specific validation of LAMP amplicons in real-time optomagnetic detection of Dengue serotype 2 synthetic DNA
}

\author{
Gabriel Antonio S. Minero ${ }^{a}$, Catarina Nogueira ${ }^{b}$, Giovanni Rizzi $^{\mathrm{a}}$, Bo Tian $^{\mathrm{c}}$, Jeppe Fock ${ }^{\mathrm{a}}$, Marco \\ Donolato $^{b}$, Mattias Strömberg ${ }^{c}$, and Mikkel F. Hansen ${ }^{a}$
}

\begin{abstract}
We report on an optomagnetic technique optimised for real-time molecular detection of Dengue fever virus under ideal as well as non-ideal laboratory conditions using two different detection approaches. The first approach is based on the detection of the hydrodynamic volume of streptavidin coated magnetic nanoparticles attached to biotinylated LAMP amplicons. We demonstrate detection of sub-femtomolar Dengue DNA target concentrations in the ideal contaminationfree lab environment within $20 \mathrm{~min}$. The second detection approach is based on sequence-specific binding of functionalised magnetic nanoparticles to loops of LAMP amplicons. Melting studies reveal that true positive and spurious amplicons have different melting points and this allows us to discriminate between them. This is found to be in a good agreement with subsequent studies on real-time sequence-specific discrimination of LAMP amplicons. The specific binding causes clustering of magnetic nanoparticles via binding to multiple sites (loops) emerging in the elongation phase of LAMP. Formation of nanoclusters is monitored via the depletion of the optomagnetic signal due to free nanoparticles. After sequence-specific validation, we claim detection of down to $100 \mathrm{fM}$ of Dengue target after 20 min of LAMP with a contamination background.
\end{abstract}

\section{Introduction}

Loop-mediated isothermal amplification (LAMP) is a powerful single-tube isothermal nucleic acid amplification technique yielding billions of copies from less than 10 copies of input template (DNA or RNA) within 1 hour. ${ }^{1}$ In this technique, a template with a length of about 200 nucleotides is converted into a dumbbell structure with single-stranded loops to which primers hybridise and are extended under isothermal conditions. ${ }^{1-3}$ Due to the isothermal operation and high sensitivity, LAMP is promoted for molecular diagnostics in peripheral laboratories of developing countries. ${ }^{4}$

Routine end-point detection of LAMP amplicons can be performed by gel electrophoresis, ${ }^{2}$ by visual inspection of turbidity, ${ }^{3}$ or via the colour of a magnesium-sensitive indicator. ${ }^{4-6}$ The LAMP technique has been implemented on a disk ${ }^{7}$ and in a lateral flow device. ${ }^{8}$ Reports have emerged on multiplex readout of LAMP products by fluorescent probes targeting loops, ${ }^{9}$ by quenching of fluorescence from unincorporated inner primers, ${ }^{10}$ by multiple endonuclease restriction to generate fluorescence signal from the incorporated inner primers, ${ }^{11}$ and by appearance of fluorescence when quencher strands are displaced via toehold-mediated binding of DNA probes. ${ }^{12}$ DuVall et al. presented a bead-based LAMP assay on a chip that detected the inhibition of large amounts of amplicons on the aggregation of magnetic beads under chaotropic conditions. ${ }^{13}$ Recently, we reported on a nanoparticle-based approach using susceptometric $^{14}$ and optomagnetic ${ }^{15}$ readouts where LAMP amplicons bound to magnetic nanoparticles (MNPs) via biotinylated primers were detected as an increase in the hydrodynamic size of the MNPs. In Ref. 15, the optomagnetic detection of Newcastle virus disease RNA was performed after mixing MNPs with a fraction of the sample after completion of the LAMP reaction. In Ref. 14, the susceptometric detection of Zika virus oligonucleotide was performed with the MNPs present in the reaction mixture during amplification, but the measurement was performed at end-point due to the long measurement time $(15 \mathrm{~min})$ and the lack of integration of temperature control with the readout system.

The use of LAMP to generate large amounts of a desired product is a double-edged sword. ${ }^{16,17}$ Among the challenges of LAMP are: (1) complex primer design, (2) assay optimization (choice of enzyme, magnesium concentration, ${ }^{14}$ additives, temperature, etc.), and (3) cross-contamination. Failure to amplify under optimum conditions may lead to generation of multiple undefined and unwanted products and even to the exclusion of the desired product. It is recommended to not open reaction vials ${ }^{1}$ near the lab where LAMP samples are prepared and to terminate the reaction at $80^{\circ} \mathrm{C}$ after a certain threshold time. ${ }^{4}$ Even an optimised assay requires avoiding leakage during LAMP, $^{1}$ because accumulation of ampliconcontaining aerosols in the lab environment causes false-positive signals. Maintaining extremely high standards of cleanliness (separation of labs, changing lab coats, etc.) is required in order to avoid occurrence of spurious (parasitic) amplicons. Research groups maintaining all of the mentioned precautions achieved specific amplification of pathogens with limits of detection (LODs) down to a few copies of, for example, $1 \mathrm{aM}$ of Dengue fever virus ${ }^{5}$ and Zika virus. $^{8}$

Recently, modifications of the LAMP technique to relax the above strict requirements have been introduced to enable LAMP to be performed in a single lab with lower requirements on contamination by amplicons from previous LAMP processes. These are typically based on the fact that the products formed from such amplicons are subject to accumulation of mutations outside the regions targeted by the primers. For example, Bst polymerase used in LAMP is estimated to introduce about one error every 5500 bases. Such order of fidelity is also claimed for the error prone Taq DNA polymerase used in the gold standard PCR. ${ }^{18}$ Amplicons can be discriminated, for example, via sequence-specific validation of single-stranded loops. ${ }^{9}$ For more efficient validation, toehold strand displacement mechanisms can be applied, ${ }^{12,19}$ which can further improve sensitivity via conformational recycling of the targeted 
sequence. ${ }^{16}$ Using a two-step readout based on catalysed hairpin assembly, the Ellington group demonstrated specific detection of a model template with an LOD of 6 copies after $1.5 \mathrm{~h} .{ }^{16}$ Further, with the use of a "protected" fluorescent probe complementary to one of the loops in a simpler assay format, they demonstrated real-time detection of 20 copies ( $1 \mathrm{aM}$ ) of target with a threshold time of about 2 h. $^{12}$

Analysis made by J. Qian et $a .^{20}$ revealed that polymerases have a higher affinity for purine oligonucleotides and hence that GA-rich templates may promote non-specific amplification. Betaine reduces the dependence of DNA melting on base composition. ${ }^{21}$ Therefore, it is often used in amplification assays to reduce stability of mismatched priming. For exponential amplification reactions (EXPAR) suffering from similar cross-contamination constraints, blocking of spurious amplicons by the DNA mismatch repair protein MutS prevents strand displacement on such templates and hence reduces spurious amplification. ${ }^{22} \mathrm{~A}$ single-stranded binding protein may reduce mismatched hybridisation by a similar mechanism. ${ }^{23}$ Recently, K. Hsieh et al. demonstrated a way to suppress "parasitic" amplification in LAMP by use of UTP instead of TTP in the LAMP cocktail and by introducing a pre-amplification UTP digestion step with heat-labile uracil-DNA-glycosylase. ${ }^{24,25}$

The detection of the product of an exponential amplification assay can be done at the end-point after a fixed amplification time or in real-time during the amplification. In the former case, the answer is typically "yes" or "no" depending on whether the amplicon signal exceeds the detection threshold. In the latter case, a dosedependent signal can be determined from the threshold time at which the signal becomes detectable. The exponential amplification makes these techniques very sensitive to a variation in detection time. ${ }^{26}$ Compared to end-point detection, real-time monitoring of the amplicon growth during LAMP provides a more accurate dosedependent analysis.

In this work, we chose Dengue Serotype 2 synthetic DNA (D2) as a model target for real-time optomagnetic detection of LAMP amplicons. Following work of Lau et $a l^{5}{ }^{5}$, we used a set of primers targeting conservative $3^{\prime}$-NCR (non-coding region) of D2 genome. Hu et al. demonstrated amplification and differentiation of Dengue virus serotype 1-4 targeting corresponding genomic regions coding non-structural proteins NS2A (D1), NS4B (D2), NS4A (D3) and 3'untraslated region (D4). ${ }^{27}$ Multiplexed LAMP with sequence-specific detection of all four Dengue serotypes in a single tube was shown in Dauner et al. ${ }^{28}$

The World Health Organization reported the Dengue virus as major public-health concern throughout tropical and sub-tropical regions of the world..$^{29}$ Every year, 50 to 100 million people are infected and at least 30,000 people die. Clinical requirements concerning sensitivity of early Dengue diagnostics were reported as $10^{6} \mathrm{pfu} / \mathrm{ml}$ in serum ${ }^{30}$ and $10^{3}-10^{6}$ RNA copies $/ \mathrm{ml}$ in serum. ${ }^{5}$ Successful trial on Dengue diagnostics in non-ideal conditions was demonstrated by Abd El Wahed et al., however, implemented using a different amplification technique. ${ }^{31}$

Here, we first demonstrate real-time measurements of the size increase of MNPs with attached amplicons using an optomagnetic technique during LAMP (detection approach I). Under ideal laboratory working conditions we obtain a sub-femtomolar LOD with a threshold time of $20 \mathrm{~min}$. Next, we investigate an alternative detection strategy (detection approach II) based on capture probes targeting the loops of the LAMP products. We present real-time monitoring of melting of DNA duplexes (bridges) formed between capture probes (on MNPs) and looped LAMP amplicons and show that such measurements can be used to discriminate between true and spurious LAMP amplicons. Finally, we employ this discrimination in a real-time detection format that detects the depletion of free MNPs as they bind to true positive LAMP amplicons and form clusters.

Using sequence-specific clustering of MNPs on the amplicons, we demonstrate a $100 \mathrm{fM}$ LOD of Dengue DNA with an assay time of $20 \mathrm{~min}$ in a single laboratory, which has been used extensively for LAMP amplification and with a spurious LAMP amplicon background of $100 \mathrm{fM}$. The presented research contributes to better understanding and tailoring of LAMP steps towards diagnostics of such severe diseases as Dengue Fever in real lab conditions.

\section{Materials and Methods}

\section{Optomagnetic setup}

The basic working principle of the optomagnetic technique as implemented by us has been reported elsewhere. ${ }^{15,32-34}$ In brief, the optomagnetic method relies on the coupled magnetic and optical anisotropies of $100 \mathrm{~nm}$ MNPs as well as of MNP clusters. The technique probes the $2^{\text {nd }}$ harmonic modulation of light transmitted through a suspension of MNPs in response to a magnetic field $B(t)=B_{0} \sin (2 \pi f t)$ applied along the light path. PMMA rods guided the light from the light source to the chip and from the chip to the photodetector. The photodetector voltage vs. time was measured using a data acquisition card and the cosine and sine components of the signal modulation at $\mathbf{2} \boldsymbol{f}$ were found in LabView using a fast Fourier transformation algorithm. The novelty of the setup in the present study compared to our previous work ${ }^{15,35}$ lies in the integration of real-time optomagnetic measurements with double-sided top and bottom heaters of a plastic chip with light access via the chip side (Fig. S1). In the present setup, we used light emitting diodes with a wavelength of $470 \mathrm{~nm}$ and a magnetic field with $\boldsymbol{B}_{\mathbf{0}}=0.8 \mathrm{mT}$. A full sweep of the frequency of the magnetic field measuring 41 points between $1 \mathrm{~Hz}$ and $2800 \mathrm{~Hz}$ was recorded in $40 \mathrm{~s}$. For further details on this setup, see Section S1 of the electronic supplementary information (ESIt).

The relevant data for the present study is the real component of the $2^{\text {nd }}$ harmonic of the transmitted light intensity, $\boldsymbol{V}_{2}^{\prime}$, which gives the component of the signal proportional to $\sin (4 \pi \boldsymbol{f} t)$. Individual MNPs with a size of $100 \mathrm{~nm}$ give rise to a negative signal in $V_{2}^{\prime}$ with a peak positioned at $\boldsymbol{f}_{\text {peak }} \approx \boldsymbol{f}_{\mathrm{B}} / \sqrt{\mathbf{3}}^{34}$, where the Brownian relaxation frequency $f_{\mathrm{B}}$ is given by

$$
f_{\mathrm{B}}=\frac{k_{\mathrm{B}} T}{\pi^{2} \eta D_{\mathrm{h}}^{3}}
$$

Here, $\boldsymbol{k}_{\mathrm{B}} \boldsymbol{T}$ is the thermal energy, $\boldsymbol{\eta}$ is the viscosity of the liquid, and $\boldsymbol{D}_{\mathbf{h}}$ is the hydrodynamic MNP diameter. Clusters of MNPs above a 
certain size interact differently with the light and display a signal of opposite sign (positive peak), which is shifted to lower frequencies due to the larger hydrodynamic size of the MNPs. Thus, clustering of MNPs results in depletion of the negative signal from single MNPs at high frequencies and in the occurrence of a positive signal in $V_{2}^{\prime}$ at low frequencies.

\section{Chip design and fabrication.}

Custom single-use and disposable polymethylmethacrylate (PMMA) chips were fabricated using a CAD design and a $\mathrm{CO}_{2}$-laser (Mini 18, 30W, Epilog, USA). Rectangular PMMA sheets with thicknesses of $0.5 \mathrm{~mm}, 2 \mathrm{~mm}$, and $0.5 \mathrm{~mm}$ were joined together using pressure sensitive adhesive (PSA, 90106, Adhesive Research, Ireland). In addition to alignment holes, the PMMA sheets contained inlet holes in the top layer and a chamber (volume of about $75 \mu$ ) in the middle layer made by the $\mathrm{CO}_{2}$-laser. The PSA was laminated on the middle layer, to which the top and bottom layers were bonded. The chips were laser cut from the bonded PMMA structures. See Section S1, ESIt for more information.

\section{Magnetic nanoparticles}

All studies were performed with multicore MNPs with a nominal mean diameter of $100 \mathrm{~nm}$ and a streptavidin surface (BNF-Starch streptavidin, prod. code 10-19-102, Micromod Partikeltechnologie $\mathrm{GmbH}$, Germany). These MNPs have a remanent magnetic moment and we have previously established a clear link between their optical and magnetic anisotropies. ${ }^{34}$ For studies with prefunctionalised particles, the streptavidin MNPs were functionalised with biotinylated DNA probes (Table S1, ESIT) according to a previously published protocol to have approximately 100 probes/MNP ${ }^{33}$ after which they were resuspended in Tris buffer with $0.1 \%$ Tween-20. All experiments were performed with a final MNP concentration of $0.1 \mathrm{mg} / \mathrm{ml}$ (detection approach I) or 0.05 $\mathrm{mg} / \mathrm{ml}$ (detection approach II). An MNP mass concentration of 0.1 $\mathrm{mg} / \mathrm{ml}$ corresponded to an MNP number concentration of about $100 \mathrm{pM}$.

\section{LAMP reaction setup}

There are four to six primers that each play a different role in LAMP: two outer primers, two inner primers (FIP, BIP) and, optionally, looped primers (LF, LB). The inner primers have extended 5 '-tails: in addition to anti-sense sequences (used for priming in the first stage) they contain 5 '-terminal sense sequences for self-priming in the later stages (Fig. 1). ${ }^{2,3}$ The self-priming and strand displacement eliminate the need for thermocycling and thus make LAMP isothermal. Furthermore, amplicons, undergo elongation resulting in periodic sequence repeats. ${ }^{2}$ Figure 1 illustrates the LAMP technique on MNPs.

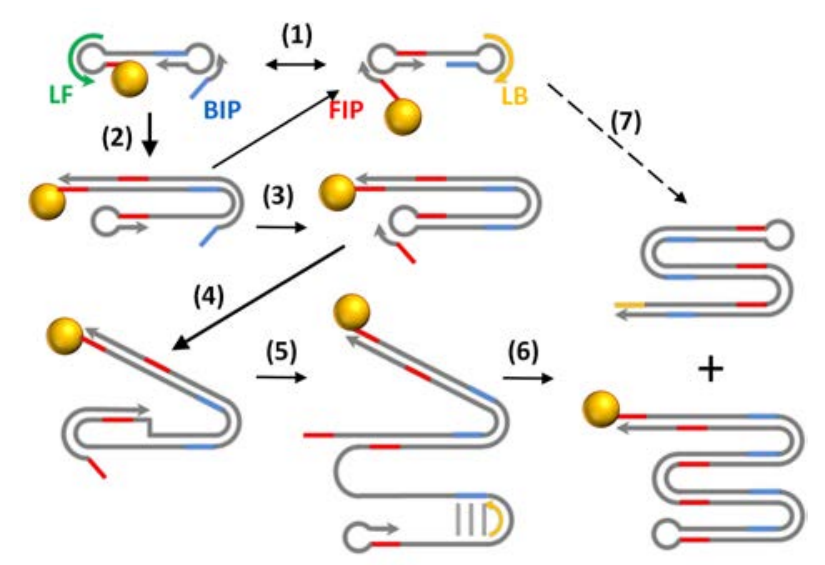

Fig. 1: Schematic and simplified illustration of LAMP on streptavidin MNPs. Amplicons are attached to streptavidin MNPs (indicated by the yellow sphere) via the biotinylated forward inner primer (FIP). (1) Autonomous replication of dumbbell structures, (2) extension of internally self-annealed $3^{\prime}$-end as well as annealing and extension of BIP at the same time, (3) extension of internally self-annealed 3 '-end and annealing of MNP-free FIP, (4) extension of FIP with strand displacement, (5) hybrid structure of dsDNA containing newly synthesised strand and ssDNA of doubled length, (6) extension of internally self-annealed $3^{\prime}$-end as well as LB (7).

All reagents for LAMP reaction were purchased from New England Biolabs, USA. DNA primer sequences were purchased from LGC Biosearch Technologies, Denmark. The LAMP reaction mixture was reconstituted from commercial LAMP buffer supported with $4 \mathrm{mM}$ magnesium sulfate, $1.4 \mathrm{mM}$ each of dNTP (deoxyribonucleotide triphosphates), Bst 3.0 polymerase, primers with the sequences adapted from Ref. 5 (Table S1, ESIT), and millipore water (18.2 $\Omega$ ).

For detection approach I, the LAMP cocktail was supported with 1.6 $\mu \mathrm{M}$ biotinylated FIP (5'-biotin), $1.6 \mu \mathrm{M}$ BIP, $0.4 \mu \mathrm{M}$ LF and LB, and $0.2 \mu \mathrm{M} \mathrm{F3}$ and B3. For detection approach II, the LAMP cocktail was supported with $1.6 \mu \mathrm{M}$ FIP and BIP, $0.2 \mu \mathrm{M}$ LB, and $0.2 \mu \mathrm{M}$ F3 and B3. Instead of LF, a biotinylated loop validation probe (LV, $\mathbf{3}^{\prime}$ biotin) was used. More details are described in the corresponding detection sections. All concentrations refer to the final values in the mixture.

For detection experiments, the D2 target (Integrated DNA Technologies, USA, Section S2, ESIt) or water (for the no template control, NTC) was added to the mixture to achieve the desired final target concentration. Negative control (NC) experiments without Bst 3.0 polymerase were also performed to check stability of the MNPs in the LAMP cocktail.

\section{Detection approach I: Capture of amplicons with bio- tinylated forward inner primers on streptavidin MNPs}

In the first approach, the forward inner primer (FIP) was biotinylated such that LAMP amplicons would attach to the streptavidin MNPs (Fig. 1). Normally, no clustering of MNPs was observed, except for a few cases where primer homodimers were formed and detected.

All pre-amplification pipetting steps were performed in a laminar air flow (LAF) bench in a lab separated from the detection lab. All components were aliquoted in the LAF bench prior to amplification 
experiments and stored at $-20^{\circ} \mathrm{C}$ until use where they were reconstituted in the LAF bench.

To perform the experiments, $60 \mu \mathrm{l}$ of the LAMP mixture was mixed with $10 \mu \mathrm{l}$ of streptavidin MNPs $(1 \mathrm{mg} / \mathrm{ml})$ and $30 \mu \mathrm{l}$ aliquots of the $\mathrm{D} 2$ target made by serial dilution of the $10 \mathrm{nM}$ stock solution. After sample loading, chips were sealed using PSA, transported to the detection lab and the liquid was centrifugally driven into the detection chamber. The chips were immediately placed inside the measurement setup at $40^{\circ} \mathrm{C}$. Upon initiation of the optomagnetic measurements, the temperature of the heaters was increased to $67^{\circ} \mathrm{C}$ where it was kept for the duration of the experiment (50 min) during which a spectrum was recorded every $40 \mathrm{~s}$. In each $\boldsymbol{V}_{2}^{\prime}$ spectrum, the position $\boldsymbol{f}_{\text {peak }}$ of the peak was found automatically in Matlab using parabolic fitting near the peak to trace $\boldsymbol{f}_{\text {peak }}$ (and hence $\boldsymbol{D}_{h}$ ) vs. time. The assay was performed at least twice for all target concentrations as well as for the no target control samples.

\section{Detection approach II: Amplicon induced sequence- specific clustering of functionalised MNPs}

In the second approach, a loop validation (LV) detection probe targeting the loop (Table S1, ESI $\mathbf{T}$ ) was introduced to enable specific detection of the LAMP amplicons formed from the D2 target. First, melting studies were performed to identify conditions at which discrimination between true positive LAMP amplicons and spurious amplicons could be obtained. Second, using these conditions, the dose-response curve for real-time detection of true positive LAMP amplicons under contaminated laboratory conditions was investigated.

\section{DNA melting studies}

LAMP products were prepared by mixing $30 \mu \mathrm{l} 2$ target (100 fM, $300 \mathrm{fM}$, and $1 \mathrm{pM}$ ) with $70 \mu \mathrm{l}$ LAMP mixture and performing the LAMP reaction in a sealed Eppendorf tube in a thermostat at $66^{\circ} \mathrm{C}$ for $1 \mathrm{~h}$. After an enzyme inactivation step $\left(10 \mathrm{~min}\right.$ at $\left.80^{\circ} \mathrm{C}\right), 5 \mu \mathrm{l}$ of LV-functionalised MNP solution $(1 \mathrm{mg} / \mathrm{ml}$ ) was added. Then, the mixture was loaded into a chip, which was sealed and placed in the measurement setup at $30^{\circ} \mathrm{C}$. After $10 \mathrm{~min}$ of incubation, a temperature sweep was performed by increasing the temperature at $0.01^{\circ} / \mathrm{s}$ while continuously recording optomagnetic spectra $(40 \mathrm{~s}$ per spectrum). The values of $\boldsymbol{f}_{\text {peak }}$ (and hence $\boldsymbol{D}_{\mathbf{h}}$ ) in the spectra were obtained by parabolic fitting and plotted vs. temperature.

\section{Dose-response curve}

All pre-amplification pipetting steps as well as the amplification and detection were performed in a single lab (the detection lab) with no special precautions. The laboratory amplicon contamination level from spurious LAMP amplicons was estimated to be equivalent to $100 \mathrm{fM}$ of D2 target DNA (Section S3, ESIT). To perform the reaction, $65 \mu \mathrm{l}$ of the LAMP mixture was mixed with $5 \mu \mathrm{l}$ of LVfunctionalised MNP solution $(1 \mathrm{mg} / \mathrm{ml})$ and $30 \mu$ aliquots of the $\mathrm{D} 2$ target made by serial dilution from the $10 \mathrm{nM}$ stock solution. Chips were loaded with samples, sealed and placed in the setup at $40^{\circ} \mathrm{C}$.
Upon initiation of the optomagnetic measurements, the temperature of the heaters was increased to $67^{\circ} \mathrm{C}$ where it was kept for the duration of the experiment ( $25 \mathrm{~min}$ ). During this time, a spectrum was recorded every $40 \mathrm{~s}$

In order to monitor sequence-specific clustering of MNPs through depletion of the signal from single MNPs, the average optomagnetic signal in the frequency range $50-500 \mathrm{~Hz}, \bar{V}_{2}^{\prime}$, was obtained and plotted vs. time. The assay was performed at least trice for NTC samples as well as for each target concentration in the range of $50-$ $1000 \mathrm{fM}$

\section{Results and Discussion}

\section{Detection approach I: Capture of amplicons with bio- tinylated forward inner primers on streptavidin MNPs}

The ratio of the FIP concentration $(1.6 \mu \mathrm{M})$ and the MNP concentration $(\approx 100 \mathrm{pM})$ was chosen such that the streptavidin MNPs were quickly saturated with the biotinylated FIP. As the LAMP amplicon self-primes during the reaction, we expect that at most one MNP would be attached to each amplicon during LAMP amplification. Therefore, the progress of the LAMP reaction could be monitored via the increase of the hydrodynamic size of the MNPs (decrease of $\boldsymbol{f}_{\text {peak }}$ ) vs. time. Figure 2A shows the $\boldsymbol{V}_{2}^{\prime}$ spectra measured vs. time for a D2 target concentration of $100 \mathrm{fM}$. The spectra are observed to show a distinct peak, which is shifted from $\boldsymbol{f}_{\text {peak }} \approx 300 \mathrm{~Hz}\left(\boldsymbol{D}_{\mathbf{h}} \approx 130 \mathrm{~nm}\right)$ to $\boldsymbol{f}_{\text {peak }} \approx 30 \mathrm{~Hz}\left(\boldsymbol{D}_{\mathbf{h}} \approx 300 \mathrm{~nm}\right)$ over the $50 \mathrm{~min}$ reaction time. This observation confirms the above hypothesis and shows that real-time optomagnetic measurements can probe the effective size of the MNPs with attached LAMP amplicons vs. time during the reaction. In addition to the shift of the peak position in Fig. 2A, we observed a reproducible drop in the $V_{2}^{\prime} / V_{0}^{\prime}$ level in the spectra. We attribute this drop to partial cosedimentation of functionalised MNPs with magnesium salts formed during the LAMP reaction.

Figure 2B shows the values of $\boldsymbol{f}_{\text {peak }}$ extracted from real-time measurements during LAMP reactions with the indicated concentrations of D2 target. Measurements on NTC samples were performed before and after performing the measurements on the dilution series. As expected for exponential amplification, a target dilution caused a delay in the response (threshold) time, but it did not change the slope of $\boldsymbol{f}_{\text {peak }}$ vs. time. The hydrodynamic size at the end-point $(20 \mathrm{~min})$ was found to correlate with the target concentration, such that a larger size was obtained for a higher target concentration. This is consistent with previous results on end-point optomagnetic detection of amplicons originating from Newcastle disease virus. ${ }^{15}$ 

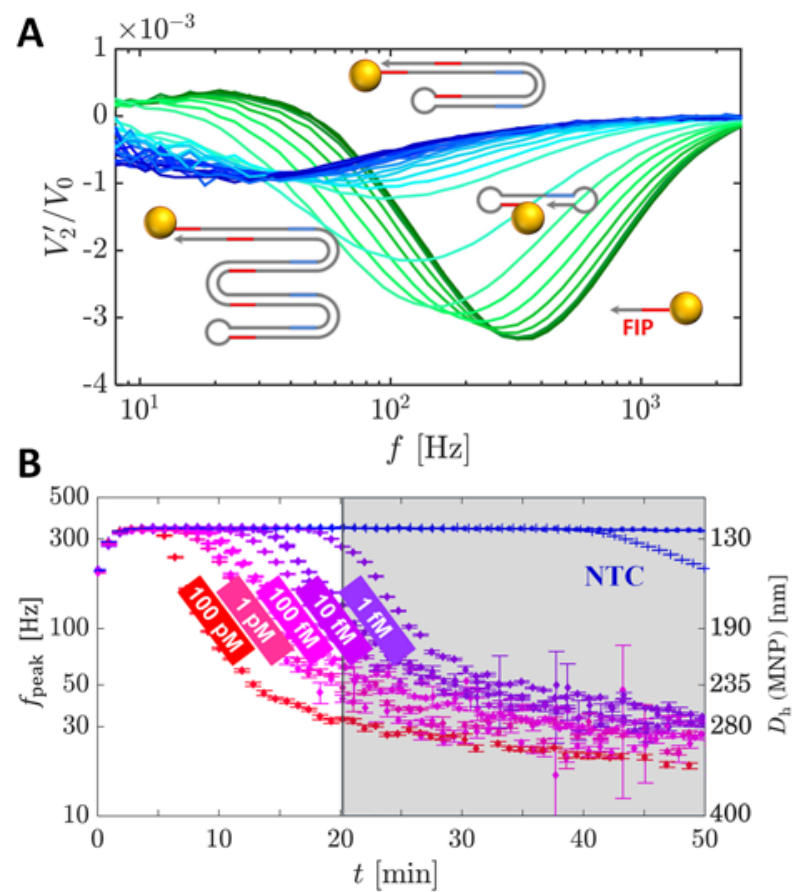

Fig. 2: Optomagnetic detection of $D 2$ amplicons in the elongation phase of LAMP. (A) $V_{2}^{\prime}$ spectra recorded vs. time (dark green to blue) during the LAMP reaction (one spectrum every $40 \mathrm{~s}$ ) for a target concentration of $100 \mathrm{fM}$. (B). Peak position $\left(f_{\text {peak }}\right)$ measured vs. amplification time for the indicated target concentrations. NTC samples were measured before and after the positive samples. The vertical line at 20 min indicates the threshold time for detection of $1 \mathrm{fM}$ target.

While the signal response vs. time depended on the target input concentration (Fig. 2B), the change in threshold time obtained for a 10 -fold dilution was at most $3 \mathrm{~min}$. Real-time monitoring of LAMP allowed us to detect this difference in the threshold times as well as to track the occurrence of false-positive signals. The NTC sample run in the clean lab prior to LAMP amplification on positive samples showed a completely flat curve, whereas the NTC sample run after the experiments on the positive samples showed a signal after about $45 \mathrm{~min}$. This tendency was further pronounced after repeated experiments (Section S3t). The above results show detection of sub-femtomolar concentrations of the target under conditions close to "ideal" as a sensitive Yes/No result, which fulfils the clinical requirements for early diagnostics of Dengue. ${ }^{30}$

It is often difficult to determine the source of contamination. In this work, we used custom built PMMA-made chips that were sealed manually after loading a sample. Therefore, we could not exclude emission of aerosols containing LAMP amplicons from the chips. The contamination level in the laboratory environment kept increasing to a level corresponding to the signal from a $100 \mathrm{fM}$ target. Therefore, only target concentrations higher than $100 \mathrm{fM}$ could be discriminated from spurious amplicons in LAMP. This is a drawback of using a biotinylated forward inner primer (FIP) since the FIP is usually extended even if the amplicon contains mutations that tend to accumulate downstream in the sequence. Therefore, the impact of sequence specificity of FIP priming on the final outcome in LAMP is low, even though LAMP is triggered by six primers.

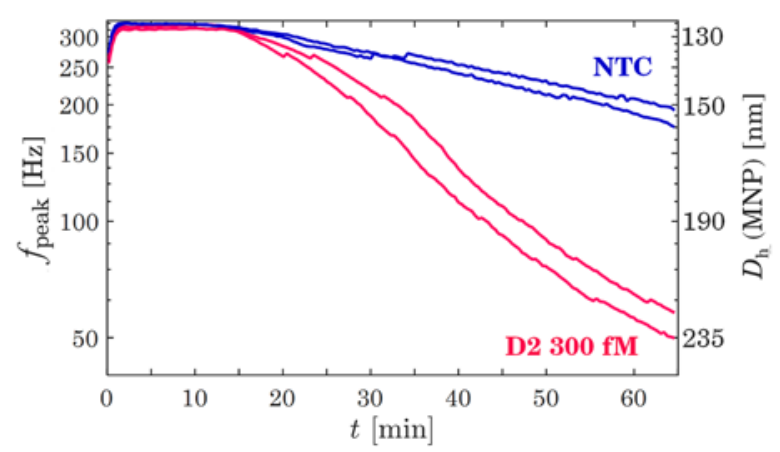

Fig. 3: Alternative optomagnetic detection of true positive (D2) and spurious (NTC) amplicons on MNPs using MNPs functionalised with the LF primer. Concentration of target in the true positive samples was $300 \mathrm{fM}$ while the estimated concentration of spurious amplicons in the NTC samples was about $100 \mathrm{fM}$ (Section S3, ESIt). Results of two repeated experiments are shown.

Along this line, we also tested the effect of biotinylating the $3^{\prime}$ end of the LF primer instead of the FIP (Fig. 3). The results showed a reaction that progressed more slowly (Fig. 3) but that also showed higher specificity and ability to distinguish between the NTC corresponding to $100 \mathrm{fM}$ target concentration and the positive sample. For the FIP-MNPs, the slope of $\boldsymbol{f}_{\text {peak }}$ vs. LAMP time was the same for spurious amplicons and the dilution series of D2 target. For the LF-MNPs, however, inhibition of LAMP was observed for the NTC (low slope of $\boldsymbol{f}_{\text {peak }}$ vs. time) compared to the $300 \mathrm{fM}$ D2 sample (steep slope vs. time). This can be observed by comparing to Fig. 2B, where the only difference between $100 \mathrm{fM}$ and $1 \mathrm{pM}$ target concentrations is an offset in the threshold time of about $3 \mathrm{~min}$. The higher specificity of the LF-MNPs can be explained by the fact that the $\mathbf{L F}$ and $\mathbf{B F}$ primers are displaced from mismatched binding sites during LAMP, without being extended. Biotinylation of the LF primer instead of the FIP resulted in better discrimination because of its shorter length (LF) and, therefore, lower probability to form homodimers.

Compared to our previous work ${ }^{15}$ the presented approach I eliminates need for post-amplification capture of amplicons on MNPs and is, therefore, less error-prone, less labour-intensive, and can be used for both real-time measurement and end-point detection.

\section{Detection approach II: Amplicon induced sequence- specific clustering of functionalised MNPs}

Here, first we demonstrate the evidence for accumulation of mutations in the spurious amplicons using DNA melting analysis. Then, we demonstrate real-time sequence validation in an asymmetric LAMP reaction without the $\mathbf{L F}$ primer. This is followed by dose-response analysis and discussion. We note that this approach has a design and underlying molecular mechanistics, which significantly differs from previous studies with MNP-based detection of LAMP products. ${ }^{14,15}$

\section{DNA melting analysis}


To search for a readout strategy with better ability to discriminate between true positive LAMP amplicons and mutation-containing spurious LAMP amplicons, following Li et al. ${ }^{16}$ we designed a $3^{\prime}$ biotinylated DNA probe to target one of the emerging loops of the LAMP amplicons. We speculate that such a DNA probe would not participate in any polymerase-mediated extension due to an MNP blocking the $3^{\prime}$-end (Section S2, ESIt). For this, a loop-validating (LV) probe (Table S1, ESIt) was used instead of the LF primer in the LAMP cocktail and we investigated whether it was possible to find conditions where we could discriminate between the binding of LV probes to loops of true positive and spurious LAMP amplicons, respectively (Section S4, ESIt).

We therefore conducted LAMP in a true positive sample and a contaminated NTC sample and studied the binding properties between MNPs with the attached probe (LV) and the amplicons true positive and spurious, respectively - through loop recognition (Fig. 4). Upon addition of LV-functionalised MNPs (LV-MNPs) we observed within minutes for both samples a shift of $\boldsymbol{f}_{\text {peak }}$ from about $300 \mathrm{~Hz}$ to $10 \mathrm{~Hz}$ (Fig. 4A) corresponding to an increase of $\boldsymbol{D}_{\mathrm{h}}$ from about $130 \mathrm{~nm}$ to about $400 \mathrm{~nm}$. This change was due to attachment of amplicons to the MNPs.
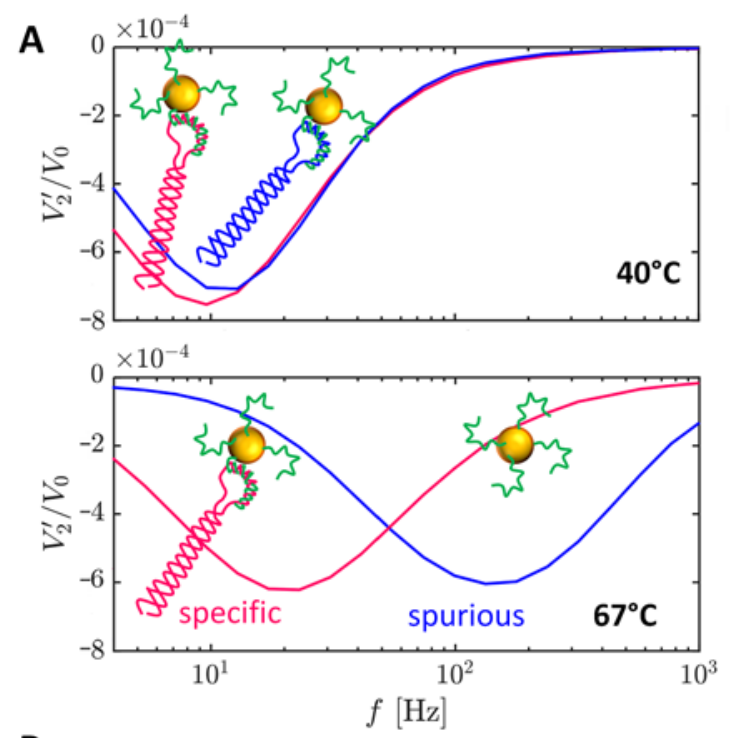

B

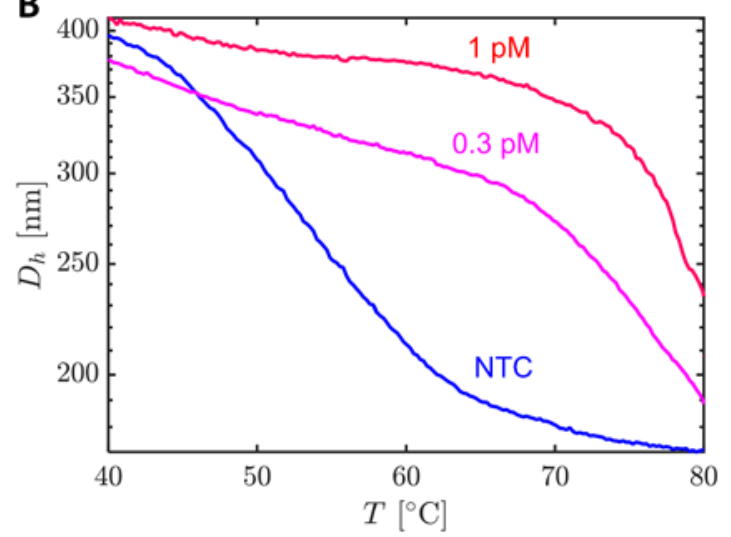

Fig. 4: Sequence-specific discrimination of true and false amplicons via LV capture probes on MNPs detected by DNA melting. (A) End-point sequencebased detection of $1 \mathrm{pM}$ Dengue target based on loop recognition: at $40^{\circ} \mathrm{C}$ (top panel) and at $67^{\circ} \mathrm{C}$ (bottom panel). (B) Melting plots $\left(0.01^{\circ} \mathrm{C} / \mathrm{s}\right.$ ) of samples containing $1 \mathrm{pM}$ target (red) and no template (blue) incubated with LV-MNPs after LAMP. Examples of the corresponding optomagnetic spectra are given in Fig. S3, ESIt.

Subsequently, we performed real-time measurements during a temperature ramp to study the melting behaviour of the MNPamplicon conjugates. The corresponding $\boldsymbol{V}_{2}^{\prime}$ spectra reflected a decreasing hydrodynamic volume with increasing temperature due to melting of loop-LV probe hybrids. The melting temperatures for the true positive and spurious amplicons attached to the capture probe via loops differed by more than $20^{\circ} \mathrm{C}$ (Fig. 4B). The best discrimination was observed at temperatures in the range $65^{\circ} \mathrm{C}$ $70^{\circ} \mathrm{C}$. No change in the optomagnetic signal intensity was observed (compare Fig. 2A and Fig. S3At).

The obtained melting trends are consistent with the accumulation of base errors in spurious amplicons through its constant recycling in LAMP, which can also be observed by endonuclease restriction analysis. ${ }^{17}$ These mutations cause the large observed difference in melting temperatures $\left(>20^{\circ} \mathrm{C}\right)$ between spurious and true positive amplicons.

\section{Dose-response analysis of real-time data}

In these studies, all pipetting steps were performed in the same laboratory with no special precautions concerning crosscontamination by amplicons from past LAMP reactions. Sequencespecific validation of the LAMP products was employed by use of LV-MNPs for the readout in a real-time LAMP reaction at $67^{\circ} \mathrm{C}$, where the melting studies showed negligible binding of LV-probes to spurious amplicons (Fig. 4).

Optomagnetic spectra measured in real-time during LAMP on true positive samples (Fig. 5A) revealed a decrease of the negative peak at about $250 \mathrm{~Hz}$ from single LV-MNPs and the occurrence of a positive peak at low frequencies $(1-20 \mathrm{~Hz})$. Corresponding experiments on the NTC sample (Fig. 5B) showed only a small decrease of the intensity of the peak from single MNPs. Following our previous reports, ${ }^{32,33}$ the observations on the true positive sample are indicative of clustering of MNPs. Each MNP binds to a loop as illustrated in Fig. 5C. The observation of clustering of LVMNPs shows that several LV-MNPs are attached to each amplicon. Thus, the results show that each amplicon exposes several binding sites (loops) during the amplification. 

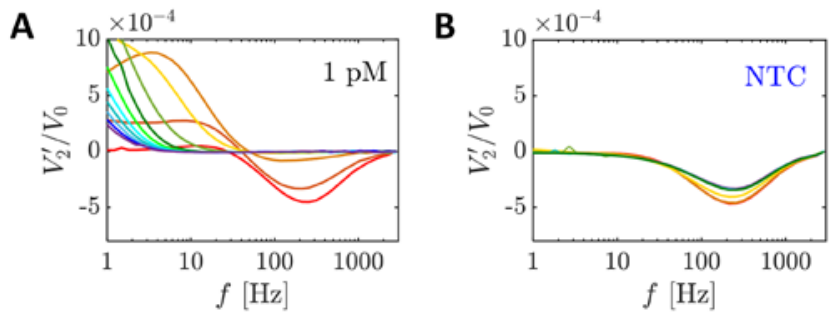

C

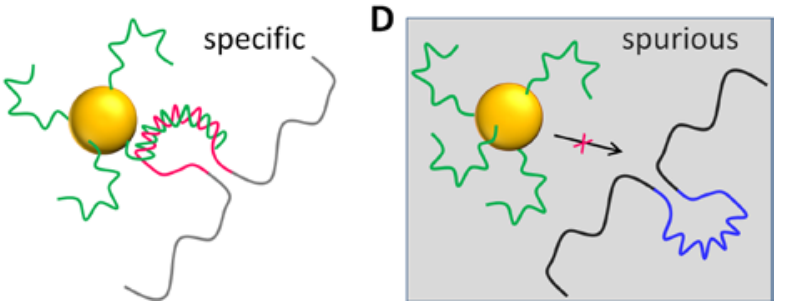

Fig. 5: Sequence-specific validation of loops in LAMP amplicons. (A)-(B) Optomagnetic spectra obtained at $67^{\circ} \mathrm{C}$ at different time points (red to blue) during LAMP reflecting multiple loops per amplicon and clustering in the case of (A) true-positive amplicons and (B) an NTC sample containing spurious amplicons. For more details, see Fig. S4, ESIt. (C)-(D) Schematic illustration of loops in specific and spurious amplicons targeted by LV probes attached to MNPs.

As a result of the clustering, the optomagnetic spectra display depletion of single MNPs to different extent depending on the target concentration in the LAMP mixture. As the relevant (and simplest) signal for the dose-response analysis for a target concentration $c$, we therefore use the signal from free MNPs at time $\boldsymbol{t}(\mathrm{min})$ after initiation of the LAMP reaction, $\overline{\boldsymbol{V}}_{\mathbf{2}}^{\prime}(\boldsymbol{c}, \boldsymbol{t})$, obtained as the average of the $V_{2}^{\prime}$-values in the range $100-500 \mathrm{~Hz}$. To eliminate any influence of a small variation in the MNP concentration, we normalise the signal at time $\boldsymbol{t}$ with that obtained at time $\boldsymbol{t}=0$ where no LAMP products have formed such that we only study the relative depletion of free MNPs. Figure 6A shows the signal $\overline{\boldsymbol{V}}_{\mathbf{2}}^{\prime}(\boldsymbol{c}, \boldsymbol{t}) / \overline{\boldsymbol{V}}_{\mathbf{2}}^{\prime}(\boldsymbol{c}, \mathbf{0})$ vs. time during real-time LAMP for the indicated target concentrations. Figure $6 \mathrm{~B}$ shows the end-point value, $\overline{\boldsymbol{V}}_{\mathbf{2}}^{\prime}(\boldsymbol{c}, \mathbf{2 0} \mathbf{\mathrm { min }}) / \overline{\boldsymbol{V}}_{\mathbf{2}}^{\prime}(\boldsymbol{c}, \mathbf{0})$, vs. target concentration obtained from triplicate experiments. Using the $3 \sigma$ criterion, we estimate an LOD of $100 \mathrm{fM}$ of Dengue target DNA under the employed non-ideal conditions.
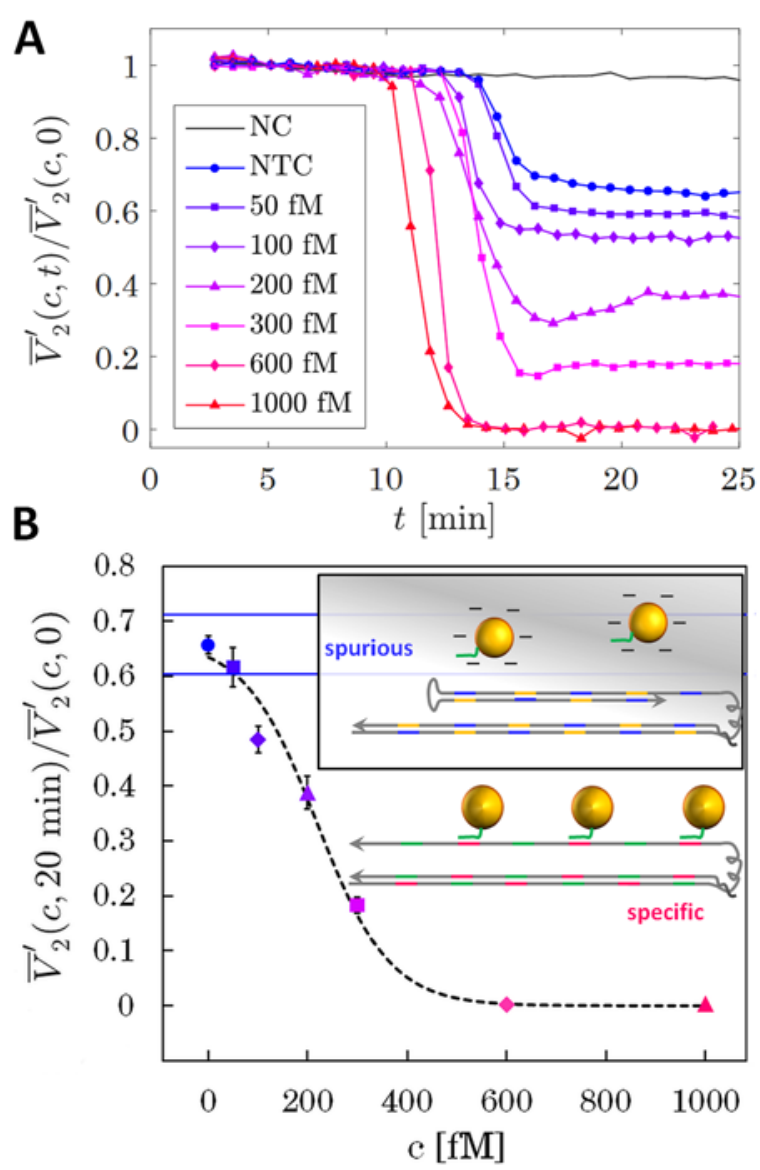

Fig. 6: Optomagnetic detection of nanoclusters in LAMP. (A) Real-time monitoring of depletion of single MNPs for different target concentration in LAMP. The normalised average $(100-500 \mathrm{~Hz})$ signal $\overline{\boldsymbol{V}}_{\mathbf{2}}^{\prime}(\boldsymbol{c}, \boldsymbol{t}) / \overline{\boldsymbol{V}}_{\mathbf{2}}^{\prime}(\boldsymbol{c}, \mathbf{0})$ corresponding to single MNPs. (B) End-point value, $\overline{\boldsymbol{V}}_{2}^{\prime}(\boldsymbol{c}, \mathbf{2 0} \mathbf{\mathrm { min }}) / \overline{\boldsymbol{V}}_{\mathbf{2}}^{\prime}(\boldsymbol{c}, \mathbf{0})$, corresponding to the single, non-depleted MNPs. The dashed line is a fit of the logistic function $y=0.67(6)\left[1-\left(1+10^{\left(c_{0}[\mathrm{fM}]-c[\mathrm{fM}]\right)^{p}}\right)^{-1}\right]$ to the dose-response curve with $\boldsymbol{c}_{\mathbf{0}}[\mathbf{p M}]=\mathbf{2 1 9}(28) \mathrm{fM}$ and $\boldsymbol{p}=\mathbf{0 . 0 0 6 0}(\mathbf{1 0})$. Numbers in parentheses indicate the uncertainty obtained from the fitting. The two blue lines show the NTC signal and the NTC signal minus $3 \sigma$ used to calculate the LOD $(n=3)$. The inset illustrates the elongation phase of LAMP for specific and spurious amplicons with and without MNP clustering, respectively.

A minor depletion of MNPs took place also for the NTC sample (Figs. 5B and 6). We speculate that this loss of MNPs in the NTC sample is caused by sedimentation of MNPs because of attachment of the capture oligonucleotides to magnesium pyrophosphate. A similar phenomenon was observed using detection approach I (Fig. 2A). At the end of an experiment, we observed an orange precipitate made from magnesium pyrophosphate and fraction of MNPs at the bottom of the chip.

The above experiments were performed with LV-probes that were biotinylated at the $3^{\prime}$-end. When using MNPs functionalised with LFprimers biotinylated at the $5^{\prime}$-end, the looped primer was inserted into the amplicon leading to a peak shift in $\boldsymbol{V}_{2}^{\prime}$ spectra in both NTC and D2 templated reactions (Fig. S5, Section S5, ESIt). However, MNP clusters were formed only in response to amplification of D2 target as in the previously described case, but the clusters were unstable because of LF 3 '-end extension and displacement of MNPs. 


\section{Discussion}

Recently, we demonstrated automated optomagnetic detection of dengue fever that took just 8 minutes, required $6 \mu$ l of serum sample and showed a limit of detection of $25 \mathrm{ng} / \mathrm{ml}$ with an upper detection range of $20000 \mathrm{ng} / \mathrm{mL}^{35}$ In that work, clustering took place because of affinity of non-structural protein (NS1) to MNP-bound monoclonal antibodies against NS1. The present work differs by implementing a molecular amplification strategy where clustering of DNA functionalised nanoparticles is mediated by LAMP amplicons in sequence-specific manner.

The observation of clustering of LV-MNPs for true positive samples is found to be in good agreement with the mechanism of LAMP. ${ }^{2,3}$ Elongation starts when an inner primer (for example, FIP) binds to the amplicon's loop followed by unlocking of the loop and DNA displacement in the hairpin stem. Figure 1 illustrates the elongation phase in detail. A looped double-stranded structure $(\boldsymbol{L})$ undergoes unwinding of the stem during extension of the FIP (step 4). The FIP extension (step 5) results in the synthesis of a complementary strand of length $\boldsymbol{L}$ (double stranded dsDNA) and an unlocked single stranded DNA (ssDNA) of the same length. The ssDNA part of this hybrid structure has multiple periodic self-annealing sites forming loops (often called cauliflower structures) with a loop at the $3^{\prime}$ end. The periodic loops can be used for binding looped primers (for example, LB) to accelerate the LAMP reaction. ${ }^{3}$ We used them as target for amplicon validation. The 3'-terminal loop is extended in step 6 leading to a fully double-stranded amplicon of double length and displacement of the previously synthesised "short copy". Therefore, the length of amplicons in the elongation phase is doubled each time an inner primer binds to a loop on an amplicon (Fig. 1).

Regarding detection approach I, we already mentioned about the better discrimination of amplicons using a biotinylated looped primer (Fig. 3). There, excess of MNP-free LF was preventing MNP clustering. In the asymmetric LF-free version of LAMP (detection approach II), exposure of long ssDNA tracts in the elongation phase allowed us to study sequence-specific clustering of MNPs. MNPs were observed to cluster very fast when the $\mathbf{L V}$ probes hybridised to the true positive amplicons via multiple binding sites in the exposed single-stranded DNA (Fig. 6A). The LV-MNPs were observed to remain bound (see cartoon in Fig. 6B). This demonstrates that time gap between steps $\mathbf{4}$ and $\mathbf{6}$ in Fig. $\mathbf{1}$ allows for sequence-specific hybridization of functionalised MNPs to the periodic loops.

Amplification of spurious amplicons is a common problem in assays with exponential growth. ${ }^{17,26}$ Even though LAMP is triggered by the combination of four to six primers, there is a lack in the sequence specificity in the binding of the primers to its target. Additionally, the inner primers are usually very long (40-50 bases) and may form primer homodimers. Once appeared, spurious amplicons interfere with the dose-dependence of LAMP unless extra validation mechanisms are employed. Spurious amplicons increase the LOD and make nucleic acid amplification assays highly sensitive to the choice of threshold time. Aerosols containing LAMP amplicons can stay in the lab environment for many days. The $100 \mathrm{fM}$ contamination level estimated for our laboratory environment agrees well with the work of Tan et $a .^{26}$ who reported on exponential amplification of Herpes target with optical detection of gold nanoparticle clusters with detection of $1 \mathrm{pM}$ target after $4 \mathrm{~min}$. However, after additional $2 \mathrm{~min}$, identical signals were observed from NTC and $1 \mathrm{pM}$ Herpes samples. ${ }^{26}$ It is, therefore, very important to have a readout method that is not prone to detection of spurious amplicons.

Different strategies to validate the output of LAMP have been demonstrated in the literature. ${ }^{9,16}$ However, no validation of amplicons in LAMP can decrease the LOD below the background level, i.e., the concentration of spurious amplicons that compete with the target template for dNTPs. For example, in the work of $\mathrm{Li}$ et al., the obtained LOD of a few copies was on the order of the background level (less than $1 \mathrm{aM}) .{ }^{16}$ Further reducing the LOD can be pursued using betaine, ${ }^{21}$ MutS protein, ${ }^{22}$ single strand binding protein, ${ }^{23}$ or the UTP/UDG approach. ${ }^{25}$

We propose the presented optomagnetic technique as a valuable tool to validate the specificity of LAMP amplicons and note that the disposable plastic chip has the potential to be mass-manufactured by injection moulding at very low cost. The sequence-specific validation of amplicons was introduced at a stage where our laboratory environment was contaminated with spurious amplicons with a concentration estimated to $100 \mathrm{fM}$ (see Fig. S2, ESIt). The validation resulted in reproducible detection of true-positive targets at concentrations exceeding this background. Based on sequence alignment, we speculate that the designed probe will be capable of discriminating not only spurious amplicon, but also amplicons made from other serotypes (D1, D3, and D4). We also do see feasible performing multiplex amplification and detection of four Dengue serotypes by performing LAMP reactions in parallel in a multichamber chip. Further optimisation of the assay, for example elimination of spurious amplicons, ${ }^{25}$ are topics of future work.

\section{Conclusions}

We investigated an optomagnetic technique for detection of Dengue Fever virus DNA. First, detection of less than $1 \mathrm{fM}$ target was demonstrated under "ideal" laboratory conditions. This level is on the order of the required clinical sensitivity for early diagnostics of Dengue. In later stages, when the lab was contaminated with amplicons from previous reactions, we studied the specificity of amplicons via targeting of amplicon loops with a capture probe tethered to MNPs. We investigated the stability of this binding for true and false positive amplicons. A difference in $T_{\mathrm{m}}$ of more than $20^{\circ} \mathrm{C}$ allowed us to discriminate between true and false positive signal at the LAMP operating temperature. The sequence-specific validation applied in real-time optomagnetic detection of LAMP led to binding of the capture probes to several loops on single amplicons, thereby linking MNPs together to form clusters in response to positive target amplification. Target concentrations above $100 \mathrm{fM}$ were still clearly detectable in the optomagnetic signal after $20 \mathrm{~min}$ of LAMP reaction, whereas the NTC only underwent a change of optical turbidity. The sequence-specific validation approach allowed us to discriminate between true positive Dengue LAMP amplicons and spurious amplicons at the expense of a higher LOD. While we have not achieved the 
clinically relevant LOD for the detection of Dengue by this approach, we believe that the molecular validation approach used can serve as a reference that will benefit the wider isothermal amplification community. Our method benefits from real-time monitoring of the interaction between LAMP products and functionalised nanoparticles. This gives us insight into kinetics and mechanistic transformations of amplicons at the nanoscale, which helps in better understanding and tailoring of LAMP steps towards diagnostics.

\section{Acknowledgements}

We thank Michael Creagh from BluSense Diagnostics for initial drawings of the chips. This work was supported by FP7 project NanoMag (\#604448), projects MMF (\#2016-2892), DFF (\#418400121B), and USAID: AID-OAA-F-16-00089. B.T. and M.S. acknowledge Swedish Research Council Formas (Project no. 221-2012-444) for financial support.

\section{References}

1 T. Notomi, Y. Mori, N. Tomita and H. Kanda, J. Microbiol., 2015, 53, 1-5.

2 T. Notomi, H. Okayama, H. Masubuchi, T. Yonekawa, K. Watanabe, N. Amino and T. Hase, Nucleic Acids Res., 2000, 28, e63.

3 K. Nagamine, T. Hase and T. Notomi, Mol. Cell. Probes, 2002, 16, 223-229.

4 C. C. Boehme, P. Nabeta, G. Henostroza, R. Raqib, Z. Rahim, M. Gerhardt, E. Sanga, M. Hoelscher, T. Notomi, T. Hase and M. D. Perkins, J. Clin. Microbiol., 2007, 45, 1936-1940. Y. L. Lau, M. Y. Lai, B. T. Teoh, J. Abd-Jamil, J. Johari, S. S. Sam, K. K. Tan and S. AbuBakar, PLoS One, 2015, 10, e0138694. N. Tomita, Y. Mori, H. Kanda and T. Notomi, Nat Protoc, 2008, 3, 877-882.

7 S. J. Oh, B. H. Park, G. Choi, J. H. Seo, J. H. Jung, J. S. Choi, D. H. Kim and T. S. Seo, Lab Chip, 2016, 16, 1917-1926. D. Lee, Y. Shin, S. Chung, K. S. Hwang, D. S. Yoon and J. H. Lee, Anal. Chem., 2016, 88, 12272-12278. Y. Mori, T. Hirano and T. Notomi, BMC Biotechnol., 2006, 6, 3.

10 C. S. Ball, Y. K. Light, C. Y. Koh, S. S. Wheeler, L. L. Coffey and R. J. Meagher, Anal. Chem., 2016, 88, 3562-3568.

11 Y. Wang, Y. Wang, R. Lan, H. Xu, A. Ma, D. Li, H. Dai, X. Yuan and J. Xu, J. Mol. Diagnostics, 2015, 17, 392-401.

12 Y. S. Jiang, S. Bhadra, B. Li, Y. R. Wu, J. N. Milligan and A. D. Ellington, Anal. Chem., 2015, 87, 3314-3320.

13 J. A. DuVall, S. T. Cabaniss, M. L. Angotti, J. H. Moore, M. Abhyankar, N. Shukla, D. L. Mills, B. G. Kessel, G. T. Garner, N. S. Swami and J. P. Landers, Analyst, 2016, 141, 56375645.

14 B. Tian, Z. Qiu, J. Ma, T. Zardán Gómez de la Torre, C. Johansson, P. Svedlindh and M. Strömberg, Biosens. Bioelectron., 2016, 86, 420-425.
Donolato, M. F. Hansen, P. Svedlindh and M. Strömberg, ACS Sensors, 2016, 1, 1228-1234. B. Li, X. Chen and A. D. Ellington, Anal. Chem., 2012, 84, 8371-8377.

K. D. Senarath, R. B. Usgodaarachchi, V. Navaratne, A. Nagahawatte, C. D. Wijayarathna, J. Alvitigala and C. L. Goonasekara, J. Tuberc. Res., 2014, 2, 168-172.

Y. H. Zhou, X. P. Zhang and R. H. Ebright, Nucleic Acids Res., 1991, 19, 6052.

S. Bhadra, Y. S. Jiang, M. R. Kumar, R. F. Johnson, L. E. Hensley and A. D. Ellington, PLoS One, 2015, 10, e0123126. J. Qian, T. M. Ferguson, D. N. Shinde, A. J. Ramírez-Borrero, A. Hintze, C. Adami and A. Niemz, Nucleic Acids Res., 2012, 40, e87.

W. A. Rees, T. D. Yager, J. Korte and P. H. Von Hippel, Biochemistry, 1993, 32, 137-144.

Y. Mitani, A. Lezhava, Y. Kawai, T. Kikuchi, A. OguchiKatayama, Y. Kogo, M. Itoh, T. Miyagi, H. Takakura, K. Hoshi, C. Kato, T. Arakawa, K. Shibata, K. Fukui, R. Masui, S. Kuramitsu, K. Kiyotani, A. Chalk, K. Tsunekawa, M. Murakami, T. Kamataki, T. Oka, H. Shimada, P. E. Cizdziel and Y. Hayashizaki, Nat. Methods, 2007, 4, 257-262. E. Mok, E. Wee, Y. Wang and M. Trau, Sci. Rep., 2016, 6, 37837.

Y. Tang, H. Chen and Y. Diao, Sci. Rep., 2016, 6, 27605. K. Hsieh, P. L. Mage, A. T. Csordas, M. Eisenstein and H. T. Soh, Chem. Commun. (Camb)., 2014, 50, 3747-9.

E. Tan, B. Erwin, S. Dames, K. Voelkerding and A. Niemz, Clin. Chem., 2007, 53, 2017-2020.

S. Hu, M. Li, L. Zhong, S. Lu, Z. Liu, J. Pu, J. Wen and X. Huang, BMC Microbiol., 2015, 15, 265.

A. L. Dauner, I. Mitra, T. J. Gilliland, S. Seales, S. Pal, S.-C. Yang, C. Guevara, J.-H. Chen, Y.-C. Liu, T. J. Kochel and S.-J. L. Wu, Diagn. Microbiol. Infect. Dis., 2015, 83, 30-36. WHO, World Heal. Organiszation, 2012, 43.

G. A. Santiago, E. Vergne, Y. Quiles, J. Cosme, J. Vazquez, J. F. Medina, F. Medina, C. Colon, H. Margolis and J. L. Munoz-Jordan, PLoS Negl. Trop. Dis., 2013, 7, e2311. A. Abd El Wahed, P. Patel, O. Faye, S. Thaloengsok, D. Heidenreich, P. Matangkasombut, K. Manopwisedjaroen, A. Sakuntabhai, A. A. Sall, F. T. Hufert and M. Weidmann, PLoS One, 2015, 10, e0129682.

A. Mezger, J. Fock, P. Antunes, F. W. Østerberg, A. Boisen, M. Nilsson, M. F. Hansen, A. Ahlford and M. Donolato, ACS Nano, 2015, 9, 7374-7382.

G. A. S. Minero, J. Fock, J. S. McCaskill and M. F. Hansen, Analyst, 2017, 142, 582-585.

J. Fock, C. Jonasson, C. Johansson and M. F. Hansen, Phys. Chem. Chem. Phys., 2017, 19, 8802-8814.

P. Antunes, D. Watterson, M. Parmvi, R. Burger, A. Boisen, P. Young, M. A. Cooper, M. F. Hansen, A. Ranzoni and M. Donolato, Sci. Rep., 2015, 5, 16145. 